\title{
Therapeutic strategies in epithelial ovarian cancer
}

\author{
Ayako Kim ${ }^{1,2}$, Yutaka Ueda ${ }^{1 *}$, Tetsuji Naka ${ }^{2}$ and Takayuki Enomoto ${ }^{{ }^{*}}$
}

\begin{abstract}
Ovarian cancer is the most lethal gynecologic malignancy. It appears that the vast majority of what seem to be primary epithelial ovarian and primary peritoneal carcinomas is, in fact, secondary from the fimbria, the most distal part of the fallopian tube.

Treatment of epithelial ovarian cancer is based on the combination of cytoreductive surgery and combination chemotherapy using taxane and platinum. Although clear cell type is categorized in indolent type, it is known to show relatively strong resistance to carboplatin and paclitaxel regimen and thus poor prognosis compared to serous adenocarcinoma, especially in advanced stages. Irinotecan plus cisplatin therapy may effective for the clear cell adenocarcinoma.

The larger expectation for improved prognosis in ovarian carcinoma is related to the use of the new biological agents. One of the most investigated and promising molecular targeted drugs in ovarian cancer is bevacizumab, a monoclonal antibody directed against VEGF. PARP inhibitor is another one. A few recent studies demonstrated positive results of bevacizumab on progression-free survival in ovarian cancer patients, however, investigation of molecular targeting drugs in patients with ovarian cancer are still underway.
\end{abstract}

Keywords: Review, ovarian cancer, conventional treatment, novel treatment, clear cell carcinoma, bevacizumab, PARP inhibitor

\section{Background}

Ovarian cancer is the most lethal gynecologic malignancy. The origin and pathogenesis of epithelial ovarian cancer (EOC) have long been investigated but still poorly understood. Studies have shown that epithelial ovarian cancer is not a single disease but is composed of a diverse group of tumors that can be classified based on distinctive morphologic and molecular genetic features [1].

Treatment of epithelial ovarian cancer (EOC) is based on the combination of surgery and chemotherapy. Over the past three decades, surgical tumor debulking, followed by platinum-based chemotherapy is the standard treatment for advanced ovarian cancer. Although response rates and complete responses in advanced disease are $>80 \%$ and $40-60 \%$, respectively, after first-line treatment with carboplatin and paclitaxel, most patients will eventually relapse with a median progression-free survival of 18 months [2]. Intraperitoneal chemotherapy possibly improve progression-free and overall survivals

\footnotetext{
* Correspondence: ZVF03563@nifty.ne.jp; enomoto@gyne.med.osaka-u.ac.jp 'Department of Obstetrics and Gynecology, Osaka University Graduate School of Medicine, 2-2, Yamadaoka, Suita, Osaka 565-0871, Japan Full list of author information is available at the end of the article
}

(PFS and OS), however, intraperitoneal chemotherapy has not been universally accepted for at least three reasons: toxic effects, intraperitoneal treatment delivery issues and complications [3].

In this review, we first focus on the origin and pathogenesis of EOC, introducing emerging concepts of a unifying theory. Next we look at the history of treatment of EOC as well as novel treatment strategies (e.g. molecular targeted treatment).

\section{Classification of epithelial ovarian cancer}

Kurman et al. have proposed a dualistic model that categorizes various types of epithelial ovarian cancer into two groups designated type I and type II $[1,4,5]$. Type I tumors are clinically indolent and usually present at a low stage, while type II tumors exhibit papillary, grandular, and solid patterns and are highly aggressive and almost always present in advanced stage (Table 1). Type I tumors include low-grade serous, low-grade endometrioid, clear cell and mucinous carcinomas and type II include high-grade serous, high-grade endometrioid and undifferentiated carcinomas. Malignant mixed mesodermal tumors (carcinosarcomas) are included in the type
C Biomed Central

(c) 2012 Kim et al; BioMed Central Ltd.2012 This is an Open Access article distributed under the terms of the Creative Commons Attribution License (http://creativecommons.org/licenses/by/2.0), which permits unrestricted use, distribution, and reproduction in any medium, provided the original work is properly cited 
Table 1 Characteristics of type I and type II tumors

\begin{tabular}{|c|c|c|}
\hline & Type I & Type II \\
\hline Clinical features & indolent & aggressive \\
\hline \multirow{4}{*}{$\begin{array}{l}\text { Histological } \\
\text { features }\end{array}$} & low-grade serous & high-grade serous \\
\hline & $\begin{array}{l}\text { low-grade } \\
\text { endometrioid }\end{array}$ & $\begin{array}{l}\text { high-grade } \\
\text { endometrioid }\end{array}$ \\
\hline & clear cell & undifferentiated \\
\hline & mucinous & carcinosarcoma \\
\hline \multirow[t]{6}{*}{ Molecular features } & K-Ras & TP53CCNE1 \\
\hline & BRAF & \\
\hline & ERBB2 & \\
\hline & PTEN & \\
\hline & CTNNB1 & \\
\hline & PIK3CA & \\
\hline
\end{tabular}

II category because their epithelial components are identical to the pure type II carcinomas.

Type I and type II tumors have remarkably different molecular genetic features as well as morphologic differences. For example, high-grade serous carcinoma (type II tumor) is characterized by very frequent TP53 mutations (> 80\% of cases) and CCNE1 (encoding cyclin E1) amplification but rarely has mutations that characterize most type 1 I tumors such as KRAS, BRAF, ERBB2, PTEN, CTNNB1, and PIK3CA [6]. In general, type I tumors are genetically more stable than type II tumors and display a distinctive pattern of mutations that occur in specific cell types. Type II tumors which show greater morphologic and molecular homogeneity are genetically unstable and have a very high frequency of TP53 mutations. These findings suggest that these two different types of ovarian cancers develop along different molecular pathways.

In terms of origin of ovarian cancer, many of researchers and gynecologic oncologists have traditionally understood that the various different ovarian tumors are all derived from the ovarian surface epithelium (mesothelium) and that subsequent metaplastic changes lead to the development of the different cell types (Table 2). It is well known that serous, endometrioid, clear cell, mucinous and transitional cell (Brenner) carcinomas morphologically resemble the epithelia of the fallopian tube, endometrium, gastrointestinal tract or endocervix and urinary bladder, respectively. The normal epithelial cells of the ovary, however, do not show any resemblance with these tumors. An alternate theory proposes that tumors with a mullerian phenotype (serous, endometrioid and clear cell) are derived from mullerian-type tissue not mesothelium. It has been suggested that they could arise from tissues ovarian epithelial tumors are embryologically derived from the mullerian duct [7]. This mullerian-type tissue (columnar epithelium, often ciliated) forms cysts located in paratubal and paraovarian locations. According to this theory, ovarian tumors develop from these cysts, not the ovarian surface epithelium. As the tumor enlarges, it compresses and eventually obliterates ovarian tissue resulting in an adnexal tumor that appears to have arisen in the ovary.

In summary, it appears that the vast majority of what seem to be primary epithelial ovarian and primary peritoneal carcinomas are, in fact, secondary. Previous data support the view that serous tumors develop from the fimbria, the most distal part of the fallopian tube, endometrioid and clear cell tumors from endometrial tissue passing through the fallopian tube resulting in endometriosis and mucinous and Brenner tumors from transitional-type epithelium located at the tubal-mesothelial junction where the fimbria makes contact to the peritoneum.

Although the data suggesting that epithelial ovarian carcinoma arises in extra-ovarian sites and involves the ovaries secondarily are compelling, low- and high-grade serous carcinomas involve the ovaries and other pelvic and abdominal organs, such as the omentum and mesentery, much more extensively than the fallopian tubes. Similarly, although endometrioid carcinomas develop from endometriosis, which frequently involves multiple sites in the pelvis, these tumors are usually confined to the ovaries. It is likely that the predisposition for growth in the ovary is multifactorial but the precise reasons for this are unknown.

The proposed model by assigning different epithelial ovarian tumors into two categories based on clinical, morphological, and molecular genetic characteristics could serve as a framework for studying ovarian cancer pathogenesis, but this model is not complete and does not resolve all the issues. For example, clear cell carcinoma and mucinous cadenocarcinoma are classified as type I tumors, but unlike the other type I tumors clear cell and mucinous cell types are often high-grade at presentation and show relatively strong resistance to platinum-based chemotherapy. This model does not replace traditional histopathologic classification but can be

Table 2 Origin of ovarian carcinoma

\begin{tabular}{llll}
\hline & Serous & Endometrioid/Clear & Mucinous/Brenner \\
\hline Traditional theory & ovarian surface epithelium (mesothelim) & ovarian surface epithelium (mesothelim) & ovarian surface epithelium (mesothelim) \\
\hline Recent theory & fimbria & endometrial tissue (endometriosis) & tubal-mesothelial junction \\
\hline
\end{tabular}


expected to draw attention to the molecular genetic events that play a role in the tumor progression and can give light on new approaches to early detection and treatment of ovarian cancer.

\section{Conventional treatment of EOC Early disease: FIGO stage I-II}

Due to the lack of effective screening programs, ovarian cancer is diagnosed at an early stage only in about $25 \%$ of the cases. In most of these cases surgery is able to cure the disease, and the five-year survival rate for earlystage (stage I or II) ovarian cancer is around 90\% [3]. Adjuvant chemotherapy for early stage ovarian cancer is still controversial but some studies have shown its benefit under confined conditions. According to the results of two studies from the International Collaborative Ovarian Neoplasm group and the EORTC, patients with IA or IB FIGO stage, non-clear-cell histology, well-differentiated (G1) tumors, and an "optimal" surgery (performed according to international guidelines, with pelvic and retroperitoneal assessment), appear not to benefit from chemotherapy [8]. Thus, it is commonly believed that, at least in these cases chemotherapy can be probably avoided and patients can be advised to undergo clinical and instrumental follow-up. In all the other (early stage) patients (adjuvant) chemotherapy is indicated [3].

\section{Advanced disease: FIGO III-IV}

The standard treatment for patients with advanced ovarian cancer is maximal surgical cytoreduction (total abdominal hysterectomy, bilateral salpingo-oophorectomy, pelvic and para-aortic lymphadenectomy and omentectomy) followed by systemic platinum-based chemotherapy and, actually, is reasonable to expect a 5-year survival for $10-30 \%$ of women diagnosed with ovarian cancer at stage III or IV [3]. The concept of primary debulking surgery is to diminish the residual tumor burden to a point at which adjuvant therapy will be optimally effective. The percentage of patients with advanced ovarian cancer who can optimally undergo cytoreductive surgery seems to range from $17 \%-87 \%$ [9], depending on the report reviewed. This percentage can largely depend on the experience of the surgeon.

Recently, an interesting randomized control trial on treatment of advanced ovarian cancer was conducted by Vergote et al. [10]. This phase III randomized study compared primary debulking surgery followed by chemotherapy with neoadjuvant chemotherapy followed by interval debulking surgery in patients with advanced ovarian cancer (Table 3). The median overall survival was 29 months in the primary-surgery group and 30 months in the neoadjuvant chemotherapy group and this difference was not statistically significant. Also, $\mathrm{n}$ difference was observed in median
Table 3 Comparison of primary debulking surgery and neoadjuvant chemotherapy

\begin{tabular}{lcc}
\hline & $\begin{array}{c}\text { Primary debulking } \\
\text { surgery }\end{array}$ & $\begin{array}{c}\text { Neoadjuvant } \\
\text { chemotherapy }\end{array}$ \\
\hline $\begin{array}{l}\text { Number of } \\
\text { patients }\end{array}$ & 336 & 334 \\
\hline $\begin{array}{l}\text { Age: Median } \\
\text { (range) }\end{array}$ & $62(25-86)$ & $63(33-81)$ \\
\hline Stage & $257(76.5 \%)$ & $253(75.7 \%)$ \\
$\quad$ IIIC & $77(22.9 \%)$ & $81(24.3 \%)$ \\
$\quad$ IV & $2(0.6 \%)$ & $0(0 \%)$ \\
\hline PFS & 12 months & 12 months \\
\hline OS & 29 months & 30 months \\
\hline
\end{tabular}

progression-free survival. These results are thoroughly discussed among the experts in this field; it is believed that upfront maximal cytoreduction is still the standard, although further research should focus on how to select patients that cannot receive optimal cytoreduction and that can benefit from a neoadjuvant strategy. When deciding debulking surgery, we should assess predictive factors with respect to recidual macroscopic disease after debulking surgery which is the strongest independent variable in predicting survival [10].

\section{Recurrent disease}

Despite the activity of first-line chemotherapy, which gives response rates up to $80 \%$ in first line treatment, the majority of patients die of their recurrent disease [2]. Therefore, a large proportion of patients are candidates for second-line treatment. Platinum sensitivity, which is defined by a response to first-line platinumbased therapy, has been found to predict the response to subsequent retreatment with a platinum-containing regimen frequently used for salvage therapy.

In general, patients who progress or have stable disease during first-line treatment or who relapse within 1 month are considered to be 'platinum-refractory'. Patients who respond to primary treatment and relapse within 6 months are considered 'platinum-resistant', and patients who relapse more than 6 months after completion of initial therapy are characterized as 'platinum-sensitive' [11]. It is known that longer platinum free interval (PFI) increases the chances for a benefit by platinum rechallenge. This has been reported especially for PFI longer than 12 months. Patients who are relapsing 6-12 months following the end of their initial regimen may benefit less and are, usually classified as so-called 'partially sensitive' [12] (Table 4).

Several randomized trials have been performed in platinum-sensitive patients. The ICON-4/OVAR 2.2 study compared the combination chemotherapy (platinum plus paclitaxel) to single chemotherapy (platinum alone) in 802 patients with 'platinum-sensitive' relapsed ovarian 
Table 4 Association of platinum sensitivity and PFI

\begin{tabular}{|c|c|c|c|c|}
\hline Platinum sensitivity & resistant & & & \\
\hline & refractory & resistant & partially sensitive & sensitive \\
\hline$\overline{\mathrm{PFI}}$ & during/immediately after chemotherapy & $<6$ months & 6-12 months & $>12$ months \\
\hline
\end{tabular}

cancer. Results demonstrated that both survival and progression free survival were significantly longer in combination therapy compared to platinum alone [13].

The optimal treatment of patients with partially platinum-sensitive recurrent ovarian cancer is not clearly defined. Trabectedin, a marine-derived antineoplastic agent initially isolated from the tunicate Ecteinascidia turbinate, has recently been introduced to this setting of patients. This agent is currently produced synthetically and its mechanism of anti-cancer action is based on DNA minor-groove binding [14].

Patients with platinum refractory and resistant are good candidates for novel investigational approaches and studies of drug resistance. Single-agent therapy is considered the standard treatment in these patients. Low response rates are recorded in these patients with the use of topotecan, docetaxel, oral stoposide, pegylated liposomal doxorubicin (PLD), gemcitabine, ifosfamide and hexamethylmelamine. The pegylated liposomal doxorubicin (PLD), a new formulation of doxorubicin, compared with the conventional, assumes favorable pharmacokinetic properties such as a lower plasma concentration peak, lower clearance, smaller distribution volume, longer half-life and higher AUC, resulting in a different and more convenient toxicity and efficacy profile [15]. The efficacy of PLD has been clearly documented in recurrent ovarian cancer giving the rationale for its use also in the first-line setting. The MITO-2 (Multicenter Italian Trials in Ovarian cancer) phase III was designed to compare the combinations of carboplatin plus paclitaxel to an experimental arm with carboplatin plus PLD in first-line treatment of ovarian cancer patients. Results have been presented at ASCO 2010 showing that carboplatin plus PLD is not superior to carboplatin plus paclitaxel in terms of PFS; the median PFS was 19 and 16.8 months in the former and the latter arms, respectively. However, given the observed confidence interval and the different toxicity profile it has been proposed that carboplatin plus PLD could be considered an alternative to standard therapy [16].

Several randomized trials have been performed in platinum-sensitive patients. A multicenter phase III study, recently published, the Calypso study [12], has compared efficacy and safety of PLD-carboplatin and carboplatin-paclitaxel in 976 relapsed platinum-sensitive ovarian cancer patients. The trial showed superiority of the experimental arm in terms of PFS (11.3 months versus 9.4; $\mathrm{HR}=0.821,95 \% \mathrm{CI} 0.72-0.94 ; P=0.005)$. The safety profile of PLD-carboplatin appears remarkably different from that of carboplatin plus paclitaxel. The PLD-carboplatin combination was associated with a higher incidence of anemia and thrombocytopenia (rarely requiring transfusions) and a higher incidence of stomatitis and cutaneous toxicity (that were rarely severe, $14 \%$ of G1-2). Notably, however, the PLD-carboplatin combination was associated with a very low incidence of hair loss and neurotoxicity compared between the 2 arms was found in terms of response rate [16]. One interesting observation of this trial was in PLD-carboplatin arm compared to carboplatin-paclitaxel there was the reduction in the rate of hypersensitive reaction (grade > 2: 5.6\% versus 18.8\%) Therapeutic Strategies in Epithelial Ovarian Cancer and this is important information since hypersensitive reactions are reported in the general practice in patients treated with carboplatin up to $25 \%$.

\section{Treatment of clear cell type of EOC}

Although clear cell type is categorized in Type I (indolent) ovarian cancer, it is known to show relatively strong resistance to carboplatin and paclitaxel regimen and thus poor prognosis compared to serous adenocarcinoma (SAC), especially in advanced stages. Previously Sugiyama et al. investigated clinical characteristics of clear cell carcinoma (CCC) of the ovary and showed that patients with CCC were significantly more likely to have FIGO Stage I disease than were patients with SAC (48.5\% versus $16.6 \%$ ). However, a high recurrence rate was noted in those patients with Stage IC CCC (37\%) and the survival rates for those stage IC CCC patients were lower than those for patients with SAC. Also, the 3-year and 5-year survival rates for Stage III CCC patients were significantly lower compared with Stage III SAC patients [17].

Enomoto et al. demonstrated that clear cell or mucinous carcinoma histologic type did not respond to the carboplatin-paclitaxel combination chemotherapy (response rates $18 \%, 13 \%$, respectively compared to $81 \%$ for serous adenocarcinoma and $89 \%$ for endometrioid adenocarcinoma) [18]. Considering those previous reports, alternative chemotherapy regimens or novel treatment for clear cell and mucinous carcinoma should be investigated.

Takakura et al. performed phase II trial of paclitaxelcarboplatin therapy (TC arm) versus irinotecan plus cisplatin therapy (CPT-P arm) as first-line chemotherapy for clear cell adenocarcinoma of the ovary [19]. PFS 
showed no significant difference between the 2 treatment groups. Because there were more patients with large residual disease in the CPT-P arm, they performed a subset analysis by removing those patients, and then compared the PFS with that of patients without residual disease less than $2 \mathrm{~cm}$. The PFS tended to be longer in the CPT-P group, although the difference was not statistically significant. A phase III randomized trial of CPT-P arm versus TC arm undertaken by JGOG (Japanese Gynecologic Oncology Group) has closed and we are waiting for the results. According to a small retrospective in Japan, gemcitabine showed modest activity and is the most effective agent to clear cell adenocarcinoma of the ovary [20].

\section{History of chemotherapy regimens for EOC}

Over the years, experts and research groups have explored different combinations of antitumor drugs in order to improve the prognosis of ovarian cancer (Table $5)$. In 1976, the report by Witshaw and Kroner on the efficacy of cisplatin in ovarian cancer produced the modern era of combination chemotherapy (platinumbased combination therapy).

In the 1980 s/early 1990 another turning point in the treatment of ovarian cancer was related to the discovery of paclitaxel, and active constituent of bark of the Pacific Yew tree, Taxus brevifolia. This agent acts by promoting microtubular assembly and stabilizes tubulin polymer formation and has a great deal of activity in epithelial ovarian cancer. Two randomized trials, the GOG 111 and the OV-10, comparing cisplatin/paclitaxel with cisplatin/cyclophosphamide, showed additional clinical benefit when cyclophosphamide was replaced by paclitaxel in the first-line setting [21-23].

Carboplatin, a cisplatin analogue is reported to have fewer marked side effects, especially such toxicities as nausea, renal toxicity, hearing loss, and neuromuscular toxicities than cisplatin. The carboplatin-paclitaxel combination is now considered an almost universal regimen in the management of epithelial ovarian cancer, and with a response rate of about $65 \%$, PFS of $16-21$ months

Table 5 The history of chemotherapy regimens for ovarian cancer

\begin{tabular}{lll}
\hline Study & Chemotherapy & regimen Reference \\
\hline GOG22 & Melphalan $<$ CA & Cancer 51:783, 1983 \\
\hline GOG47 & CA $<$ CAP & Cancer 57:1725, 1986 \\
\hline GOG52 & CAP $=$ CP & JCO 7:457, 1989 \\
\hline GOG111 & CP $<$ TP & NEJM 334:1, 1996 \\
\hline OV10 & CP $<$ TP & JNCI 92:699, 2000 \\
\hline GOG158 & TP $=$ TC & ASCO 1999; \#1373, 1374 \\
\hline SCOTROC & TC $=$ DC & ASCO 2002; \#804 \\
\hline
\end{tabular}

and an OS of 32-57 months it is the standard arm in all the recent trials performed in this disease.

In the last two decades, some studies have been performed in order to improve the efficacy of first-line chemotherapy such as by delivering drugs in epithelial ovarian cancer through the intraperitoneal (IP) route.

GOG 172 phase III trial revealed a prolonged survival in the arm of intraperitoneal (IP) therapy compared to the arm of intravenous (IV) therapy (65.6 and 49.7 months respectively; $P=0.03$ ). Also PFS was better in the IP-therapy arm than in the IV-therapy group (23.8 versus 18.3 months, $P=0.05$ ) [24]. However, a significantly higher rate of both hematologic and non-hematologic toxicities, including catheter related complications was observed in the arm of IP chemotherapy in this study. In most countries the intravenous route of administration of chemotherapy is still preferred.

Some studies have investigated the possibility to substitute paclitaxel with other drugs in order to improve the efficacy of treatment and to reduce toxicities, in particular alopecia and neurotoxicity (Table 6) [25].

The first attempt to develop this strategy was performed with docetaxel, a semisynthetic taxane with pharmacologic and pharmacokinetic advantages, compared to paclitaxel. This approach was sustained by emerging evidences suggesting superiority over anthracyclines and paclitaxel in metastatic breast cancer [26,27].

In ovarian cancer, docetaxel demonstrated activity [28], both in paclitaxel-resistant patients [29], and in primary ovarian cancer, in association with carboplatin [30]. To further investigate these promising findings, the SCOTROC-1 phase III study was performed. 1077 patients with ovarian cancer were randomly assigned to receive carboplatin IV (AUC 5) plus either docetaxel at $75 \mathrm{mg} / \mathrm{m} 2$ (1-h intravenous infusion) or paclitaxel at $175 \mathrm{mg} / \mathrm{m} 2$ (3-h intravenous infusion) [31]. Contrary to the previous results from several preclinical studies, which suggested that docetaxel might be more beneficial to paclitaxel, this phase III study did not demonstrate a survival advantage for carboplatin plus docetaxel over carboplatin plus paclitaxel treatment.

Carboplatin plus paclitaxel combination was associated with higher neurotxicity than carboplatin plus docetaxel therapy. Conversely, treatment with carboplatin plus docetaxel was associated with statistically more events of G3-4 neutropenia ( $94 \%$ versus $84 \%, P<0.001$ ) and neutropenic complications than other treatment, requiring the frequent use of G-CSF support. Based on these data docetaxel with carboplatin has been considered a possible alternative to carboplatin-paclitaxel treatment in patients at very high risk of neurotoxicity, but has not replaced carboplatin-paclitaxel as standard treatment. 
Table 6 Comparative investigations of the possibility to substitute paclitaxel with other drugs

\begin{tabular}{|c|c|c|c|c|c|c|}
\hline Study & Treatment arms & FIGO stage & $\mathrm{n}$ & PFS (m) & OS(m) & $p$ \\
\hline SCOTROC-1 & & III-IV & & & & 0.71 \\
\hline & Carboplatin (AUC5)+Paclitaxel (175 mg/mq) & & 539 & 14.8 & N.A & \\
\hline & Carboplatin (AUC5)+Docetaxel $(75 \mathrm{mg} / \mathrm{mq})$ & & 538 & 15.0 & N.A & \\
\hline MITO-2 & & IC-IV & & & & N.S. \\
\hline & Carboplatin (AUC5) + Paclitaxel (175 mg/mq) & & 410 & 16.8 & 53.2 & \\
\hline & Carboplatin (AUC5) + Liposomal doxorubicin (30 mg/mq) & & 410 & 19.0 & 61.6 & \\
\hline
\end{tabular}

N.A.: not accessed

N.S.: not significant

According to a recent review article [32], gemcitabine was the most common drug used in clinical trials. Gemcitabine-based combination therapy showed an average response rate of $27.2 \%$, and was the most common therapy among the group of regimens with above average response rate and progression-free survival.

\section{Novel treatment strategies of EOC}

The larger expectation for improved prognosis in EOC is related to the use of the new biological agents. The deeper knowledge of ovarian cancer biology has led to the identification of multiple molecular targets, such as growth factor receptors, signal transduction pathways, cell cycle regulators, and angiogenic mechanisms. In this section, we overlook the major two molecular targeted agents applied to ovarian cancer treatment; anti-VEGF antibody bevacizumab and PARP inhibitor Olaparib.

\section{Bevacizumab}

One of the most investigated and promising molecular targeted drugs in ovarian cancer is bevacizumab, a monoclonal antibody directed against VEGF. VEGF expression is higher in ovarian cancer tumors than in normal ovarian tissue or benign ovarian tumors, and increasing VEGF expression in either cytosolic fractions derived from ovarian cancer tumors or serum VEGF levels in preoperative serum is considered to be associated with advanced stage and worse survival.

In order to inhibit the VEGF pathway, there are two primary strategies: (1) inhibition of the VEGF ligand with antibodies or soluble receptors and (2) inhibition of the VEGF receptor (VEGFR) with tyrosine kinase inhibitors (TKIs), or receptor antibodies. Of the VEGF targeting therapies, the most experience has been with a monoclonal antibody that binds the VEGF ligand, known as bevacizumab (Avastin). Bevacizumab is a $149-\mathrm{kDa}$ recombinant humanized monoclonal IgG1 anti-VEGF antibody. It has been FDA-1 approved for the treatment of metastatic colorectal, breast, and non-small cell lung cancer and shows promise in the treatment of ovarian cancer. Several phase II studies have shown that bevacizumab is active in recurrent ovarian cancer [33,34].
Two phase III trials (GOG218, ICON 7) have recently evaluated the role of bevacizumab in first-line chemotherapy as an adjunct to carboplatin and paclitaxel. The GOG 218 is a multicenter, placebo-controlled trial with the primary end point to determine whether the addition of bevacizumab ( $15 \mathrm{mg} / \mathrm{kg}$ every 21 days) to standard chemotherapy is able to prolong PFS after primary cytoreductive surgery. Recently bevacizumab plus chemotherapy (carboplatin-paclitaxel) and bevacizumab maintenance was demonstrated to be able to prolong PFS of about 4 months (10.3 months versus 14.1 months) compared to carboplatin-paclitaxel alone [35]. Another multicenter trial is the ICON 7, an open label, two-arm trial, enrolling patients with high risk or advanced (stage I-IV) epithelial ovarian cancer to receive carboplatin plus paclitaxel or carboplatin-paclitaxel plus bevacizumab given concurrently and as maintenance up to 18 cycles. The bevacizumab used in this trial was half of that given in the GOG 218 study. This trial also showed that the addition of bevacizumab is able to prolong PFS compared to standard carboplatin-paclitaxel [36].

Another study, OCEANS trial, showed that addition of bevacizumab prolonged PFS in platinum-sensitive recurrent ovarian carcinoma cases [37].

\section{PARP inhibitor, olaparib}

The poly (ADP-ribose) polymerases (PARPs) are a large family of multifunctional enzymes [38]. PARP-1, the most abundant isoform, plays a key role in the repair of DNA single-strand breaks through the repair of base excisions. The inhibition of PARPs leads to the accumulation of DNA single-strand breaks, which causes DNA double-strand breaks at replication forks. These doublestrand breaks are repaired in normal cells mainly by the error-free homologous recombination double-stranded DNA repair pathway, in which essential components are the tumor-suppressor proteins BRCA1 and BRCA2. In the absent of either BRCA1 or BRCA2, these lesions are not repaired, which results in cell cycle arrest and cell death, although there is an alternate pathway to nonhomologous end-joining for DBS repair [39]. 
Women with inherited mutations in BRCA1 on chromosome $17 \mathrm{q} 21$ or BRCA2 on chromosome $13 \mathrm{q} 31$ are at significantly higher risk of developing breast and ovarian cancer than women in the control population. The lifetime risks of ovarian cancer are $54 \%$ for BRCA 1 and $23 \%$ for BRCA2 mutation carriers [40]. Inherited mutations in those genes are found in $5-10 \%$ of all ovarian cancer patients. However, over $50 \%$ of high-grade serous or undifferentiated carcinomas (Type II ovarian cancer) showed loss of BRCA function, either by genetic or epigenetic events, which resulted in HR DNA repair defects [41].

The discovery of epigenetic mechanism of BRCA1/2 germinal mutation and the association of this mutation with ovarian cancer in $5-10 \%$ of the cases, led to the therapeutic concept of "synthetic lethality" [42]. In fact, in patients carriers BRCA mutation, PARP inhibition results in unrepaired DNA single-strand and double strand breaks and so cell death [43].

Fong et al. administered to fifty patients, the majority of which were platinum refractory, the PARP inhibitor olaparib with a favorable safety profile and a high response rate, in particular in patients with BRCA mutation. In patients with platinum-resistant and even platinum-refractory disease the response rate (of PARP inhibitor, olaparib) was of $41.7 \%$ and $15.4 \%$, respectively [44]. Olaparib (AZD2281) was tested in BRCA-mutated patients with ovarian, primary peritoneal, and fallopian tube cancer. In the study, 20 patients $(40 \%)$ responded to the therapy. Currently, randomized trials of olaparib and other PARP inhibitors in patients with ovarian cancer are underway.

\section{Conclusion}

Maximal surgical cytoreduction followed by systemic taxane and platinum-based chemotherapy is the standard treatment for patients with ovarian cancer. Molecular targeting therapy may improve the prognosis of them.

\section{Abbreviations \\ CA: Cyclophosphamide + Adriamycin; CAP: Cyclophosphamide + Adriamycin + Cisplatin; CP: Cyclophosphamide+ Cisplatin; TP: Paclitaxel + Cisplatin; TC: Paclitaxel + Carboplatin; DC: Docetaxel + Carboplatin \\ Author details \\ 'Department of Obstetrics and Gynecology, Osaka University Graduate School of Medicine, 2-2, Yamadaoka, Suita, Osaka 565-0871, Japan. ${ }^{2}$ Laboratory for Immune Signal, National Institute of Biomedical Innovation, Osaka, Japan.}

\section{Authors' contributions}

Dr. K wrote the manuscript, and Dr. E, Dr. U and Dr. N approved it. All authors read and approved the final manuscript.

\section{Competing interests}

The authors declare that they have no competing interests.
Received: 5 January 2012 Accepted: 13 February 2012

Published: 13 February 2012

\section{References}

1. Kurman RJ, Shih le M: The origin and pathogenesis of epithelial ovarian cancer: a proposed unifying theory. Am J Surg Pathol 2010, 34:433-443.

2. Rubin SC, Randall TC, Armstrong KA, Chi DS, Hoskins WJ: Ten-year followup of ovarian cancer patients after second-look laparotomy with negative findings. Obstet Gynecol 1999, 93:21-24.

3. Hennessy BT, Coleman RL, Markman M: Ovarian cancer. Lancet 2009, 374:1371-82.

4. Shih le M, Kurman RJ: Ovarian tumorigenesis: a proposed 1 model based on orphological and molecular genetic analysis. Am J Pathol 2004, 164:1511-1518.

5. Kurman RJ, Visvanathan K, Roden R, Wu TC, Shih le M: Early detection and treatment of ovarian cancer: shifting from early stage to minimal volume of disease based on a new model of carcinogenesis. Am J Obstet Gynecol 2008, 198:351-356.

6. Cho KR, Shih le M: Ovarian cancer. Annu Rev Pathol 2009, 4:287-313.

7. Dubeau L: The cell of origin of ovarian epithelial tumours. Lancet Oncol 2008, 9:1191, 7. Review.

8. Trimbos JB, Parmar M, Vergote I, et al: International Collaborative Ovarian Neoplasm trial and Adjuvant ChemoTherapy In Ovarian Neoplasm trial: two parallel randomized phase III trials of adjuvant chemotherapy in patients with early-stage ovarian carcinoma. J Natl Cancer Inst 2003, 95:105-112.

9. Ramirez I, Chon HS, Apte SM: The Role of Surgery in the Management of Epithelial Ovarian Cancer: Role of Surgery.[http://www.medscape.com/ viewarticle/738258_3].

10. Vergote I, Trope CG, Amant F, et al: Neoadjuvant chemotherapy or primary surgery in stage IIIC or IV ovarian cancer. N Engl J Med 2010, 363:943-953.

11. Markman M, Reichman B, Hakes T, et al: Responses to second-line cisplatin-based intraperitoneal therapy in ovarian cancer: influence of a prior response to intravenous cisplatin. J Clin Oncol 1991, 9:1801-1805.

12. Pisano C, Bruni GS, Facchini G, Marchetti C, Pignata S: Treatment of recurrent epithelial ovarian cancer. Ther Clin Risk Manag 2009, 5:421-426.

13. Parmar MK, Ledermann JA, Colombo N, et al: Paclitaxel plus platinumbased chemotherapy versus conventional platinum-based chemotherapy in women with relapsed ovarian cancer: the ICON4/AGOOVAR-2.2 trial. Lancet 2003, 361:2099-2106.

14. Monk BJ, Herzog TJ, Kaye SB, et al: Trabectedin plus pegylated liposomal Doxorubicin in recurrent ovarian cancer. J Clin Oncol 2010, 28:3107-3114.

15. Vaage J, Donovan D, Mayhew E, Abra R, Huang A: Therapy of human ovarian carcinoma xenografts using doxorubicin encapsulated in sterically stabilized liposomes. Cancer 1993, 72:3671-3675.

16. Pujade-Lauraine E, Wagner U, Aavall-Lundqvist E, et al: Pegylated liposomal Doxorubicin and Carboplatin compared with Paclitaxel and Carboplatin for patients with platinum-sensitive ovarian cancer in late relapse. J Clin Oncol 2010, 28:3323-3329.

17. Sugiyama T, Kamura T, Kigawa J, et al: Clinical characteristics of clear cell carcinoma of the ovary: a distinct histologic type with poor prognosis and resistance to platinum-based chemotherapy. Cancer 2000, 88:2584-2589.

18. T Enomoto CK, Yamasaki M, Sugita N, Otsuki $Y$, Ikegami H, Matsuzaki N, Yamada T, Wakimoto A, Murata $Y$ : Is clear cell carcinoma and mucinous carcinoma of the ovary sensitive to combination chemotherapy with paclitaxel and carboplatin? Proc Am Soc Clin Oncol 2003, 22:477s, (abstr\#1797).

19. Takakura S, Takano M, Takahashi F, et al: Randomized phase II trial of paclitaxel plus carboplatin therapy versus irinotecan plus cisplatin therapy as first-line chemotherapy for clear cell adenocarcinoma of the ovary: a JGOG study. Int J Gynecol Cancer 2010, 20:240-247.

20. Yoshino K, Enomoto T, Fujita M, Ueda Y, Kimura T, Kobayashi E, Tsutsui T, Kimura T: Salvage chemotherapy for recurrent or persistent clear cell carcinoma of the ovary: a single-institution experience for a series of 20 patients. Int J Clin Oncol 2011.

21. McGuire WP, Ozols RF: Chemotherapy of advanced ovarian cancer. Semin Oncol 1998, 25:340-348.

22. Piccart MJ, Bertelsen K, James K, et al: Randomized intergroup trial of cisplatin paclitaxel versus cisplatin-cyclophosphamide in women with 
advanced epithelial ovarian cancer: three-year results. J Natl Cancer Inst 2000, 92:699-708.

23. Muggia FM, Braly PS, Brady MF, et al: Phase III randomized study of 1 cisplatin versus paclitaxel versus cisplatin and paclitaxel in patients with suboptimal stage III or IV ovarian cancer: a gynecologic oncology group study. J Clin Oncol 2000, 18:106-115.

24. Armstrong DK, Bundy B, Wenzel $L$, et al: Intraperitoneal cisplatin and paclitaxel in ovarian cancer. N Engl J Med 2006, 354:34-43.

25. Pignata S, Cannella L, Leopardo D, Pisano C, Bruni GS, Facchini G: Chemotherapy in epithelial ovarian cancer. Cancer Lett 2011, 303:73-83.

26. Chan S, Friedrichs K, Noel D, et al: Prospective randomized trial of docetaxel versus doxorubicin in patients with metastatic breast cancer. J Clin Oncol 1999, 17:2341-2354.

27. Jones SE, Erban J, Overmoyer B, et al: Randomized phase III study of docetaxel compared with paclitaxel in metastatic breast cancer. J Clin Oncol 2005, 23:5542-5551.

28. Kaye SB, Piccart M, Aapro M, Francis P, Kavanagh J: Phase II trials of docetaxel (Taxotere) in advanced ovarian cancer-an updated overview. Eur J Cancer 1997, 33:2167-2170.

29. Rose $P G$, Blessing JA, Ball $H G$, et al: A phase II study of docetaxel in paclitaxel-resistant ovarian and peritoneal carcinoma: a Gynecologic Oncology Group study. Gynecol Oncol 2003, 88:130-135.

30. Vasey PA, Atkinson $R$, Coleman $R$, et al: Docetaxel-carboplatin as first line chemotherapy for epithelial ovarian cancer. Br J Cancer 2001, 84:170-178

31. Vasey PA, Jayson GC, Gordon A, et al: Phase III randomized trial of docetaxel carboplatin versus paclitaxel-carboplatin as first-line chemotherapy for ovarian carcinoma. J Natl Cancer Inst 2004, 96:1682-1691.

32. Matsuo K, Lin YG, Roman LD, Sood AK: Overcoming Platinum Resistance in Ovarian Carcinoma. Expert Opin Investig Drugs 2010, 19:1339-1354.

33. Ellis $L M$, Hicklin DJ: VEGF-targeted therapy: mechanisms of anti-tumour activity. Nat Rev Cancer 2008, 8:579-591.

34. Raspollini MR, Castiglione F, Garbini F, et al: Correlation of epidermal growth factor receptor expression with tumor microdensity vessels and with vascular endothelial growth factor expression in ovarian carcinoma. Int J Surg Pathol 2005, 13:135-142.

35. Burger RA, Brady MF, Bookman MA, Fleming GF, Monk BJ, Huang H, Mannel RS, Homesley HD, Fowler J, Greer BE, Boente M, Birrer MJ, Liang SX: Gynecologic Oncology Group. Incorporation of bevacizumab in the primary treatment of ovarian cancer. N Engl J Med 2011, 365:2473-83.

36. Perren TJ, Swart AM, Pfisterer J, Ledermann JA, Pujade-Lauraine E, Kristensen G, Carey MS, Beale P, Cervantes A, Kurzeder C, du Bois A, Sehouli J, Kimmig R, Stähle A, Collinson F, Essapen S, Gourley C, Lortholary A, Selle F, Mirza MR, Leminen A, Plante M, Stark D, Qian W, Parmar MK, Oza AM: ICON7 Investigators. A phase 3 trial of bevacizumab in ovarian cancer. N Engl J Med 2011, 365:2484-96.

37. Aghajanian C, Finkler NJ, Rutherford T, Smith DA, Yi J, Parmar H, Nycum LR, Sovak MA: J Clin Oncol 2011, 29, Memorial Sloan-Kettering Cancer Center, New York, NY; Florida Hospital Gynecologic Oncology, Florida Hospital Cancer Institute, Orlando, FL; Yale University School of Medicine, New Haven, CT; Northwest Cancer Specialists, Vancouver, WA; Genentech Inc., South San Francisco, CA; Forsyth Regional Cancer Center, WinstonSalem, NC. OCEANS: A randomized, double-blinded, placebo-controlled phase III trial of chemotherapy with or without bevacizumab (BEV) in patients with platinum-sensitive recurrent epithelial ovarian (EOC), primary peritoneal (PPC), or fallopian tube cancer (FTC). (suppl; abstr LBA5007).

38. Rouleau M, Patel A, Hendzel MJ, Kaufmann SH, Poirier GG: PARP inhibition: PARP1 and beyond. Nat Rev Cancer 2010, 10:293-301.

39. Itamochi H: Targeted therapies in epithelial ovarian cancer: Molecular mechanisms of action. World I Biol Chem 2010, 1:209-220.

40. King MC, Marks JH, Mandell JB: Breast and ovarian cancer risks due to inherited mutations in BRCA1 and BRCA2. Science 2003, 302:643-646,

41. Press JZ, De Luca A, Boyd N, et al: Ovarian carcinomas with genetic and epigenetic BRCA1 loss have distinct molecular abnormalities. BMC Cancer 2008, 8:17.

42. Helleday T: The underlying mechanism for the PARP and BRCA synthetic lethality: clearing up the misunderstandings. Mol Oncol 2011, 5:387-93.

43. Fong PC, Boss DS, Yap TA, et al: Inhibition of poly(ADP-ribose) polymerase 1 in tumors from BRCA mutation carriers. N Engl J Med 2009, 361:123-134.
44. Fong PC, Yap TA, Boss DS, et al: Poly(ADP)-ribose polymerase inhibition: frequent durable responses in BRCA carrier ovarian cancer correlating with platinum-free interval. J Clin Oncol 2010, 28:2512-2519.

doi:10.1186/1756-9966-31-14

Cite this article as: Kim et al:: Therapeutic strategies in epithelial ovarian cancer. Journal of Experimental \& Clinical Cancer Research 2012 31:14.

\section{Submit your next manuscript to BioMed Central and take full advantage of:}

- Convenient online submission

- Thorough peer review

- No space constraints or color figure charges

- Immediate publication on acceptance

- Inclusion in PubMed, CAS, Scopus and Google Scholar

- Research which is freely available for redistribution

Submit your manuscript at www.biomedcentral.com/submit 